\title{
Asymmetric Passive Dynamic Walker
}

\author{
Craig Honeycutt, John Sushko, and Kyle B. Reed \\ Department of Mechanical Engineering \\ University of South Florida \\ Tampa, FL, 33620, U.S.A.
}

\begin{abstract}
The objective of this research is to better understand the dynamics of gait asymmetry in humans with central nervous system damage, such as stroke, by using a model of a passive dynamic walker (PDW). By changing the mass, mass location, knee location, and leg length of one leg while leaving the parameters of the other leg unchanged, we show that stable asymmetric walking patterns exist for PDW models. The asymmetric PDW model shows several stable walking patterns that have a single, double, and quadruple repeat pattern where the step lengths between the two legs differ by over $15 \%$. This model will allow an analysis of the passive dynamics of walking separate from the cognitive control in asymmetric human walking to test different gait rehabilitation hypotheses.
\end{abstract}

keywords: passive dynamic walker, gait rehabilitation, asymmetric gait

\section{INTRODUCTION}

This paper demonstrates the dynamics of a passive dynamic walker (PDW) model and how different asymmetric gait patterns arise when specific design parameters are changed on only one of the two legs. A PDW model is used in this sense because it has been previously shown that a PDW exhibits a repeatable human-like gait [1]. This simple model for gait enables the ability to alter and examine the dynamics of alternate gait patterns without examining the cognitive influences of a human.

The focus of this research is to demonstrate different asymmetric gait patterns and show how our model can be used to aid current and future rehabilitation methods. While this model has limitations, it can easily be applied in simple cause and effect scenarios. One scenario in particular can be seen from studying those who suffer from spasticity in their joints [2]. By tuning our model to include a velocity dependent damper at a joint, we could simulate and evaluate corrective measures to enable more efficient walking by altering other parameters, such as the height of a shoe or adding a torsional spring to one of the opposite side joints. More generally, our model allows the testing of rehabilitation methods to give a sense of their effects before enlisting a human participant.

Another reason for advancing the applications of passive dynamic walkers for gait rehabilitation is the limited amount of material published. To the best of the authors' knowledge, no other research pairs PDW's with correcting the dynamics of the human gait. In fact, the only other known non-human adaptation model for gait rehabilitation was done using a humanoid robot which adds significant complexity due to its controls [3]. In this paper we will demonstrate several asymmetric step patterns by examining each leg and its accompanying parameters throughout the PDW's natural gait cycles.

\section{BACKGROUND}

A passive dynamic walker (PDW) is a device that exhibits a steady and stable gait down a slope without any energy inputs except the forces due to gravity. A PDW has two important collision events that occur during its gait: the knee strike and the heel strike. The energy lost at these collisions is gained as the device descends along a slope. This concept parallels that of a rimless wheel, first studied by Margaria [4] and later by McGeer [1]. These models show that a rimless wheel rolling down a slope with a constant angle conserves momentum about its collision point. The wheel will eventually settle into a dynamic equilibrium just as a PDW does walking down the same slope [1]. McGeer's research paved the way for the compass gait and related passive dynamic walking models [5]. A compass gait is essentially a double pendulum model with two leg masses and a hip mass, which follow gaitlike patterns [6]. Chen extended the compass gait to include a full mathematical model with knees [7]. In this paper, we extend her model by distinguishing the left and right legs, thus allowing the design parameters to be changed so that asymmetric gaits can be examined. Passive dynamic walkers have also been extended for use in bipedal walkers using minimal amounts of energy [8].

In our parametric study, PDW's allow us to separate the purely mechanical aspects of walking from the neurological controls of the human body. This is beneficial when studying patients whose gait changes, such as those who have suffered a stroke, since we can focus solely on the effects of physical devices that could be added as a means of rehabilitation or assistance. By using a PDW instead of a humanoid robot, gait can be studied in a purely passive way. One major difference between humanoid robots and passive walking devices is the state in which they achieve equilibrium. Humanoid robots follow a quasi-static pattern where they are stable throughout each phase of motion. This pattern can be illustrated by examining the Honda ASIMO, which was a breakthrough in robot design. Passive walking on the other hand achieves a state of dynamic equilibrium where there are points in the gait that can be described as controlled free fall.

Our aim is to extend this analysis of the dynamic changes in walking to asymmetric gait patterns. As we introduce several different gait patterns, we will further our understanding of 
how these apply to those with gait impairments and eventually give insight to projects such as the Gait Enhancing Mobile Shoe (GEMS) [9][10], which aims to correct asymmetric walking patterns. The idea of the GEMS is based on recent neuroscience research, which has shown that individuals with an unstable gait are able to temporarily regain symmetric walking patterns by training on a split-belt treadmill [11][12]. Split-belt treadmills enable independent control of each leg, allowing one leg to effectively move faster than the other. This method of rehabilitation creates after effects with a symmetric walking pattern that diminishes over time and only partially transfers to walking over ground [13]. To fix the perception differences that occur when moving from a treadmill, where the surroundings are stationary, to actual walking, where the environment is moving, devices such as the GEM Shoe aim to recreate the split-belt treadmill effect in a natural walking environment where the cognitive senses of moving are also experienced. This asymmetric PDW model will allow us to test the gait changes associated with the physical changes of walking over ground, walking on a treadmill, and walking on the GEM Shoe, which is essentially walking with one leg over ground and one leg on a treadmill. The asymmetric PDW models should also allow for the testing of a large variety of gait rehabilitation methods before testing them on individuals with gait impairments.

Currently, the only study published using a non-human adaptation model for split-belt training is [3]. In that study, Otoda et al. used a bipedal walking robot and adapted it to walk on a split-belt treadmill. However, this type of robot does not truly mimic the human gait. We believe the use of an asymmetric PDW model could more accurately describe the gait involved in rehabilitation. By developing a model for an asymmetric PDW, we can gain valuable data not only of the dynamics of motion for those who suffer from gait impairment, but also the dynamic changes of going from a split-belt treadmill to solid ground. From our model we could use collected data for advancing other rehabilitation devices by providing a test bed in which rehabilitation researchers could test the mechanics of their devices without any human interaction.

\section{Mathematical Model}

A kneed passive dynamic walker can be described as an un-actuated multipendulum system, as shown in Figure 1. In our model, we differentiate between the stance leg (st) which is the leg that is in contact with the ground, and the swing leg $(s w)$, which is the leg that is swinging freely from the hip. The stance and swing legs switch between right and left leg continually through the gait pattern, which allows the device to achieve an asymmetric gait pattern. This "ideal" model can be described as four massless rods connected by three frictionless joints (the knee joints are massless) with five point masses. The masses are the hip $(m h)$, the swing leg shank $\left(m s_{s w}\right)$, the swing leg thigh $\left(m t_{s w}\right)$, the stance leg shank $\left(m s_{s t}\right)$, and the stance leg thigh $\left(m t_{s t}\right)$. The top rods are the thigh links and the bottom rods are the shank links. The shank length,

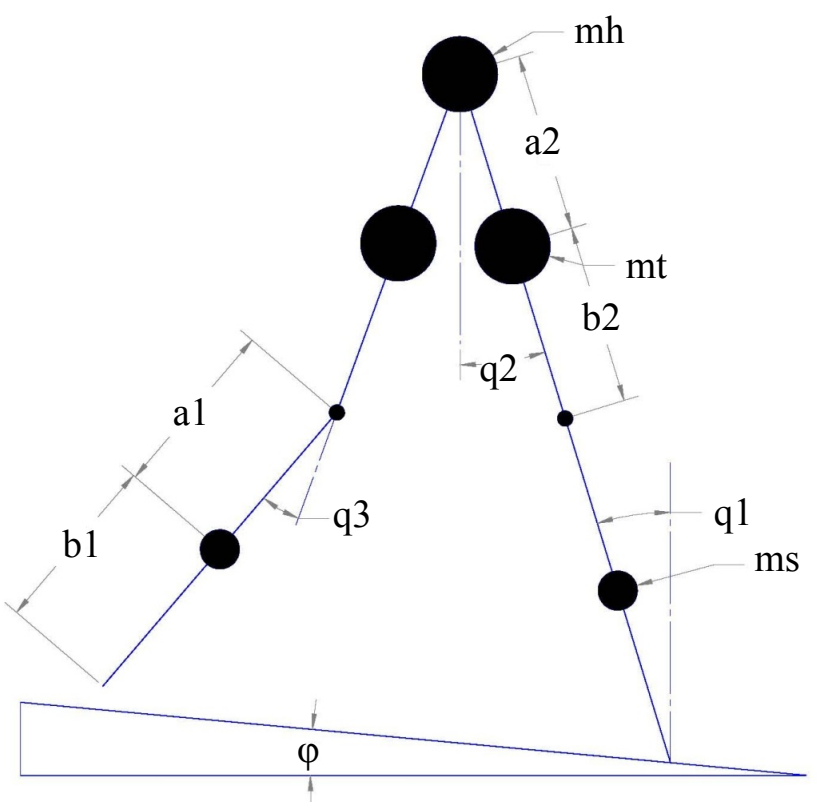

Fig. 1. Passive dynamic walker model.

$l s=a 1+b 1$, and the thigh length, $l t=a 2+b 2$, are each in terms of $s w$ or $s t$. The total length, $L=l s+l t$, is also in terms of $s t$ or $s w$.

The walker goes through two stages in its dynamics: a threelink phase and a two-link phase. The walker starts in the threelink phase where the system is a three-link pendulum. The three-link phase is described as $L_{s t}$ which is connected by the hip to $l t_{s w}$ and the knee connects $l t_{s w}$ to $l s_{s w}$. The walker remains in three-link until the knee strike event. After knee strike the knee is assumed to be locked and the system is a double pendulum. The only links in the two-link phase are $L_{s t}$ and $L_{s w}$ which are connected by the hip. The walker remains in two-link until the heel strike event. After this the $s t$ and the $s w$ switch and the walker starts the three-link stage again. The resulting gait cycle from this model is shown in Figure 2.

The dynamics for this system can be described using the Lagrangian formulation of a multi-pendulum system. Equation 1 shows the Lagrangian where $H$ is the inertia matrix, $B$ is the velocity matrix, and $G$ is the gravitational matrix:

$$
H(q) \ddot{q}+B(q, \dot{q}) \dot{q}+G(q)=0
$$

The three-link dynamics can be described by deriving the three Lagrangian matrices for a three-link pendulum system, similar to how it was done for the symmetric PDW in [7]. The matrices are as follows:

$$
\begin{gathered}
H 11=\begin{array}{c}
m s_{s t} a 1_{s t}^{2}+m t_{s t}\left(l s_{s t}+a 2_{s t}\right)^{2}+ \\
\left(m h+m s_{s w}+m_{s w}\right) L_{s t}^{2}
\end{array} \\
H 12=-\left(m t_{s w} b 2_{s w}+m s_{s w} l t_{s w}\right) L_{s t} \cos \left(q_{2}-q_{1}\right) \\
H 13=-m s_{s w} b 1_{s w} L_{s t} \cos \left(q_{3}-q_{1}\right) \\
H 21=H 12
\end{gathered}
$$




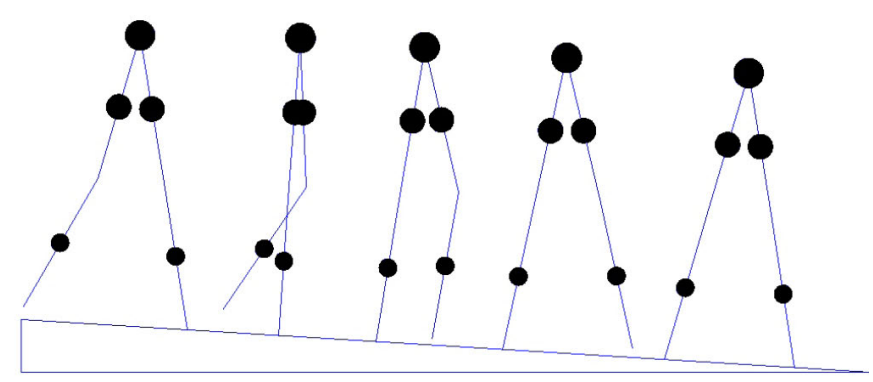

Fig. 2. The actual gait pattern of the walker with the mass location. See the attached video for a further demonstration of the passive dynamic walker asymmetric gaits with several parameters changed.

$$
\begin{aligned}
& H 22=m t_{s w} b 2_{s w}^{2}+m s_{s w} l t_{s w}^{2} \\
& H 23=m s_{s w} l t_{s w} b 1_{s w} \cos \left(q_{3}-q_{2}\right) \\
& H 31=H 13 \\
& H 32=H 23 \\
& H 33=m s_{s w} b 1_{s w}^{2} \\
& H=\left[\begin{array}{lll}
H 11 & H 12 & H 13 \\
H 21 & H 22 & H 23 \\
H 31 & H 32 & H 33
\end{array}\right] \\
& h 122=-\left(m t_{s w} b 2_{s w}+m s_{s w} l t_{s w}\right) L_{s t} \sin \left(q_{2}-q_{1}\right) \\
& h 133=-m s_{s w} b 1_{s w} L_{s t} \sin \left(q_{3}-q_{1}\right) \\
& h 211=-h 122 \\
& h 233=H 23=m s_{s w} l t_{s w} b 1_{s w} \sin \left(q_{3}-q_{2}\right) \\
& h 311=-h 133 \\
& h 322=-h 233 \\
& B=\left[\begin{array}{ccc}
0 & h 122 \dot{q}_{2} & h 133 \dot{q}_{3} \\
h 211 \dot{q}_{2} & 0 & h 233 \dot{q}_{3} \\
h 311 \dot{q}_{1} & h 322 \dot{q}_{2} & 0
\end{array}\right] \\
& g_{1}=-\left(m s_{s t} a 1_{s t}+m t_{s t}\left(l s_{s t}+a 2_{s t}\right)+\right. \\
& \left.\left(m h+m s_{s w}+m t_{s w}\right) L_{s w}\right) \sin \left(q_{1}\right) g \\
& g_{2}=\left(m t_{s w} b 2_{s w}+m s_{s w} l t_{s w}\right) \sin \left(q_{2}\right) g \\
& g_{3}=m s_{s w} b 1_{s w} \sin \left(q_{3}\right) g \\
& G=\left[\begin{array}{lll}
g_{1} & g_{2} & g_{3}
\end{array}\right]^{T}
\end{aligned}
$$

The two-link dynamics are described by the Lagrangian of a double pendulum system. The matrices are as follows:

$$
\begin{aligned}
& H 11=m s_{s t} a 1_{s t}^{2}+m t_{s t}\left(l s_{s t}+a 2_{s t}\right)^{2}+ \\
& \left(m h+m s_{s w}+m t_{s w}\right) L_{t}^{2} \\
& H 12=-\left(m t_{s w} b 2_{s w}+m s_{s w}\left(l t_{s w}+\right.\right. \\
& \left.\left.b 1_{s w}\right)\right) L_{s t} \cos \left(q_{2}-q_{1}\right) \\
& H 21=H 12 \\
& H 22=m t_{s w} b 2_{s w}^{2}+m s_{s w}\left(l t_{s w}+b 1_{s w}\right)^{2}
\end{aligned}
$$

$$
\begin{aligned}
& H=\left[\begin{array}{ll}
H 11 & H 12 \\
H 21 & H 22
\end{array}\right] \\
& h=-\left(m t_{s w} b 2_{s w}+m s_{s w}\left(l t_{s w}+b 1_{s w}\right)\right) L_{s t} \sin \left(q_{2}-q_{1}\right) \\
& B=\left[\begin{array}{cc}
0 & h \dot{q}_{2} \\
-h \dot{q}_{1} & 0
\end{array}\right] \\
& g_{1}=-\left(m s_{s t} a 1_{s t}+m t_{s t}\left(l s_{s t}+a 2_{s t}\right)+\right. \\
& \left.\left(m h+m s_{s w}+m t_{s w}\right) L_{s w}\right) \sin \left(q_{1}\right) g \\
& g_{2}=\left(m t_{s w} b 2_{s w}+m s_{s w}\left(l t_{s w}+b 1_{s w}\right)\right) \sin \left(q_{2}\right) g \\
& G=\left[\begin{array}{ll}
g_{1} & g_{2}
\end{array}\right]^{T}
\end{aligned}
$$

The collision events are derived from conservation of angular momentum about the foot for the whole system and the hip for the swing leg. For those equations and a more in depth description of the dynamics, see [7].

\section{RESUlts}

For the model to effectively exhibit an asymmetric gait, we had to change the physical parameters that defined it. Starting with an ideal (symmetric) step pattern, we took each variable and incremented it through a range of values while holding the other parameters constant. Parameters were changed on the right leg only to differ its gait pattern from the "ideal" leg. The changed parameters included: mass of the thigh, mass of the shank, location of the knee, location of the thigh mass, location of the shank mass, and total length of the leg. These parameters do not necessarily have physical analogs to those of a human, but the changed parameters generate gait patterns that may be synonymous with gaits from actual impairments.

For each parameter we changed on one leg, we recorded the step length and the limit cycle trajectory for the first 50 steps. The step length is defined as the distance between the legs at heel strike, which easily separates symmetric gait patterns from those with asymmetries. Note that our model is step based instead of time based, which allowed us to keep track of each leg as it passes from swing to stance phase. From these measures, we found that the following four step patterns emerged:

1) Symmetric step pattern: This pattern was previously described as the ideal gait. It consists of step lengths that quickly converge to one value. Its uniformity relates to a normal human gait and can be seen in Figure 3.

2) Leg specific single step pattern: This pattern consists of each leg having one unique step length and occurred when the parameters of the model were closer to the ideal values. This pattern was found by changing the location of the right shank mass, which was moved through a range of values from $0.2 \mathrm{~m}$ to $0.36 \mathrm{~m}$ measured from the end of the shank to the shank mass (a1R in Figure 1). Qualitatively, we see the greatest correlation between this gait pattern and a human asymmetrical gait pattern. For this reason, we will to look more in depth at its dynamics in the future. An example of the single step pattern is shown in Figure 4. 

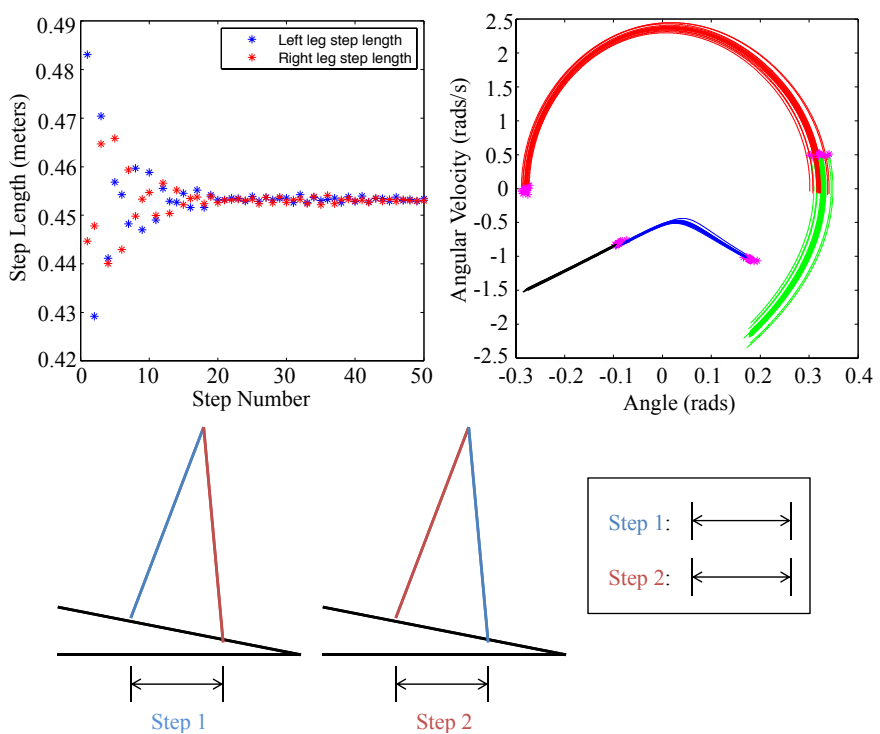

Fig. 3. Symmetric step pattern: This pattern is derived from the ideal design parameters giving it a highly stable and uniform gait. Notice that after the initial disorder, the gait pattern settles into one uniform step length.

3) Leg specific double step pattern: This pattern developed near the range of unstable cycles and can be described as each leg having two distinct step lengths. It can be seen from Figure 5 that for each step cycle one leg always has a longer step length than the other. Looking at the lower step length diagrams, it can be seen that step 2 is longer than step 1 and step 4 is longer than step 3 . This type of step pattern was seen when giving the mass of the right shank $\left(m s_{R}\right)$ values ranging from $0.032 \mathrm{~kg}$ to $0.05 \mathrm{~kg}$.

4) Leg specific quadruple step pattern: One of the most interesting gait patterns developed from our model is the quadruple step pattern, which can be described as each leg having four separate step lengths (two longer and two shorter). Following one leg through this pattern its steps are as follows: longest step, longer short step, shorter long step, and shortest step. Figure 6 more accurately depicts the described step patterns. This gait pattern was seen only once when the mass of the thigh was changed to $0.474 \mathrm{~kg}$.

Figures 3 through 6 illustrate two important characteristics needed to visualize the aforementioned step patterns; the step length and limit cycle trajectories. As seen in Figure 4 the steps settle from their initial disorder and converge to a uniform step length. Looking at Figure 5, it can be seen that there are two unique step lengths for each leg, which is the leg specific double pattern. To clarify these patterns, there are figures below the step length plots that compare each step length for each particular gait pattern. Another visualization that can be seen from the above figures is how the angular velocity varies with the angle of the right leg. These are the plots to the right and are referred to as a limit cycle trajectory plots. They accurately depict the motion of the right leg throughout
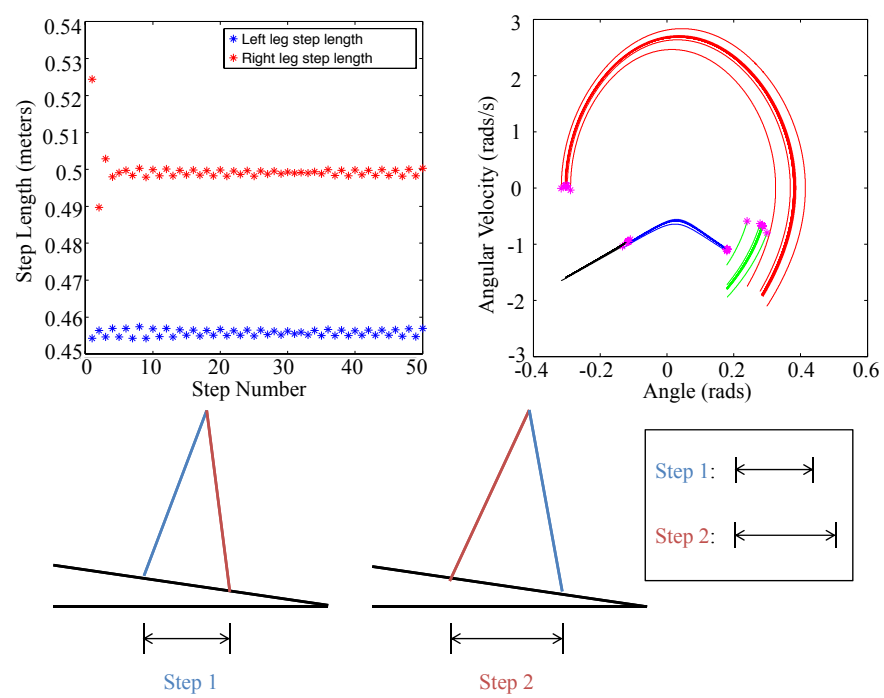

Fig. 4. Leg specific single step pattern: This pattern was found when the location of the right shank mass was in the range of $0.2 \mathrm{~m}$ to $0.36 \mathrm{~m}$. As seen from the step length plots (left) and the step length diagrams (bottom), the right leg stance step length is longer than the left leg step length. We see the most potential for this step pattern to correlate with those suffering from gait asymmetries.
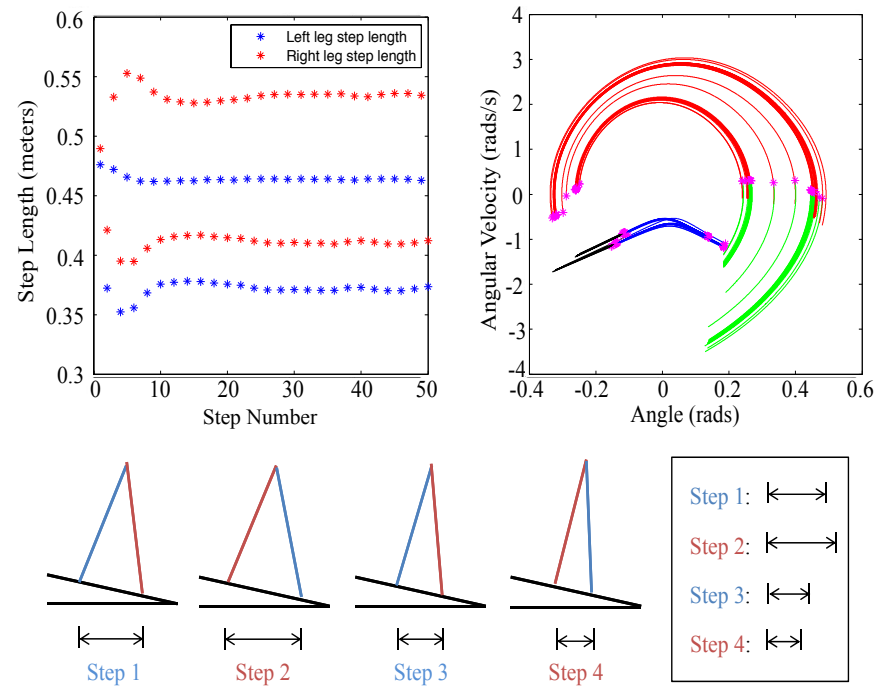

Fig. 5. Leg specific double step pattern: This pattern was found when the mass of the right shank $\left(m s_{R}\right)$ value was in the range of $0.032 \mathrm{~kg}$ to $0.05 \mathrm{~kg}$. Looking at the limit cycle trajectory plot (right) it can be seen that each stance phase has two different step trajectories. Also, from the step length plots it is clear that the right leg stance step lengths are longer than the left leg stance step lengths for each gait cycle.

each phase of their gait. In these figures, the colored curves represent the angles of the right leg through the following phases: red is the three-link left stance dynamics, green is the two-link left stance dynamics, blue is the three-link right stance dynamics, and black is the two-link right stance dynamics. Looking at these figures, the different step patterns are discerned by the location and spread of the curves. For instance, on the leg specific double step pattern trajectory, two darker curves are seen. These two curves represent the motions of each step length within the three-link and two-link phases. 

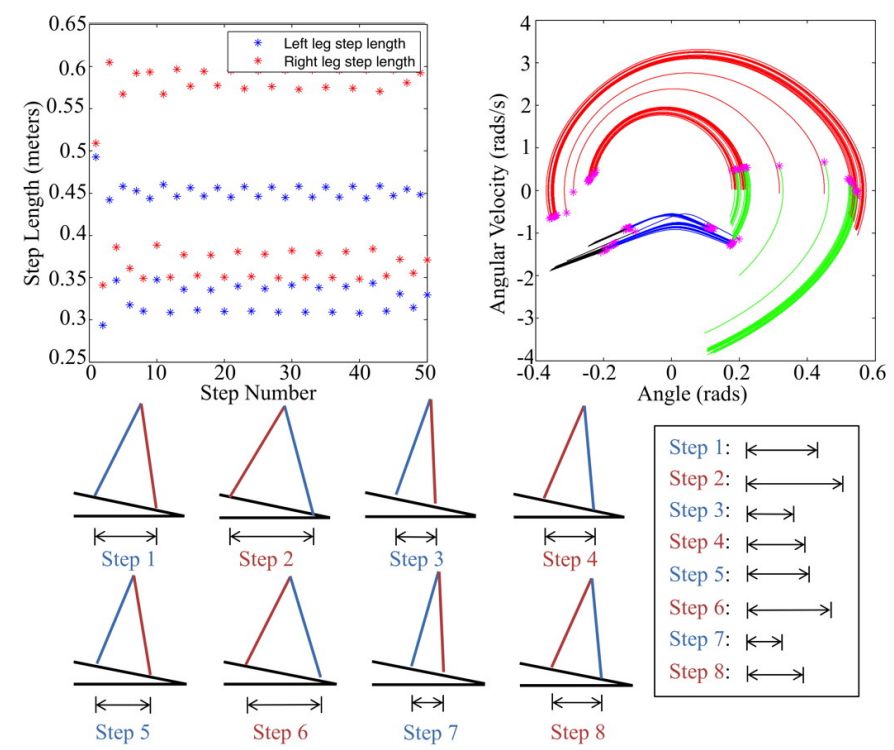

Fig. 6. Leg specific quadruple step pattern: This interesting gait pattern was found only when the mass of the right thigh $\left(m t_{R}\right)$ was given a value of $0.474 \mathrm{~kg}$. It is shown that each leg has four distinct step lengths. Looking at the bottom step length diagrams, a comparison between the step lengths for each stance phase is given.

Another interesting pattern in the limit cycle trajectories is how the graphs are shifted vertically and horizontally. As seen in Figure 4, the graph is shorter from top to bottom, but has roughly the same starting and ending angles. This indicates a shorter time span for this phase and ultimately leads to a higher angular acceleration.

By changing each design parameter and holding the others constant, we deemed the changed design parameter a passing parameter if the walker could stably walk 50 steps. A depiction of this can be seen in Figure 7. The parameter is marked high if it passed and low if it failed. Also, plotted in these figures are the left and right step lengths versus the value of the changed parameter. This plot shows the asymmetry of the gait. The ideal parameter value is located where the plots cross and is the point where the walker exhibits a symmetric gait pattern. Figures $7 \mathrm{a}$ and $7 \mathrm{~b}$ show that the mass of the shank can be stably decreased by $54 \%$ and increased by $40 \%$, yet the mass of the thigh can only be increased or decreased by $5 \%$. Changing the location of the shank mass has a larger pass range than when changing the position of the thigh mass. From this it is clear that the thigh mass is more sensitive to changes than the shank mass. Changing where the knee is located has a very large pass range. It can be located as high as the thigh mass or as low as just above the shank mass. In Figure $7 \mathrm{f}$ the larger a2R is, the closer the knee is to the shank mass. These results indicate that there are several parameters that can be changed to affect an asymmetric gait on a passive dynamic walker. None of these trials included mixing effects such as a lighter shank and a heavier thigh, but those are likely to evoke a similar range of asymmetric gaits and will be tested in future work.

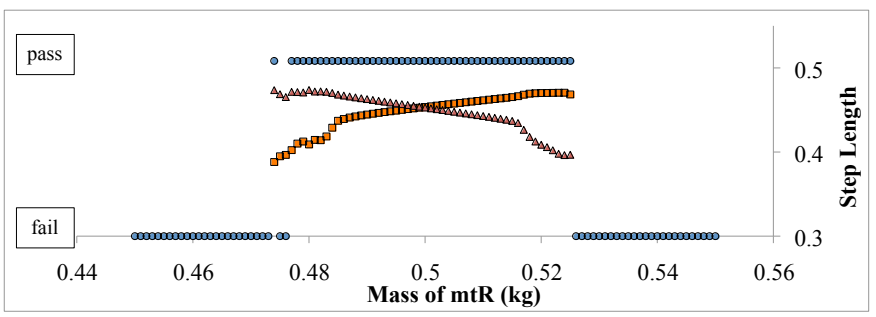

(a) Changing the mass of the right thigh.

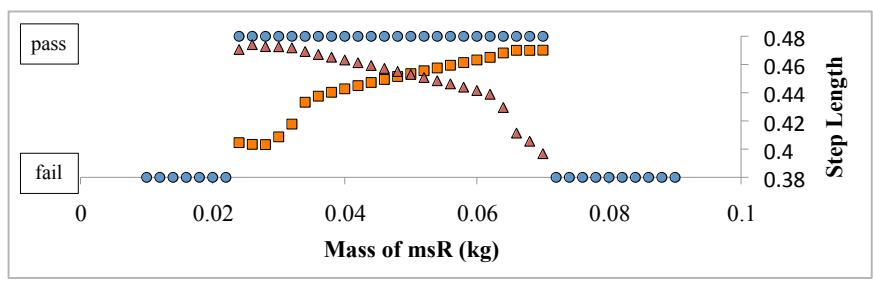

(b) Changing the mass of the right shank.

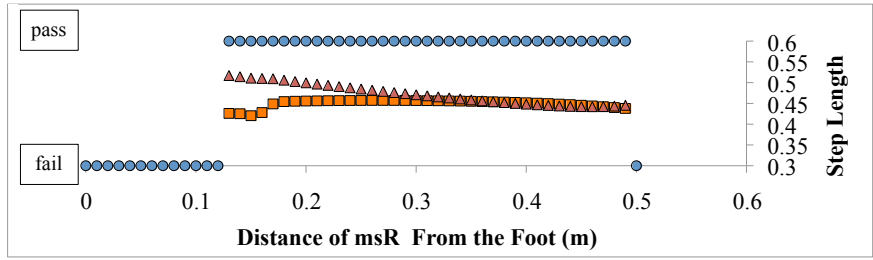

(c) Moving the mass of the right shank.

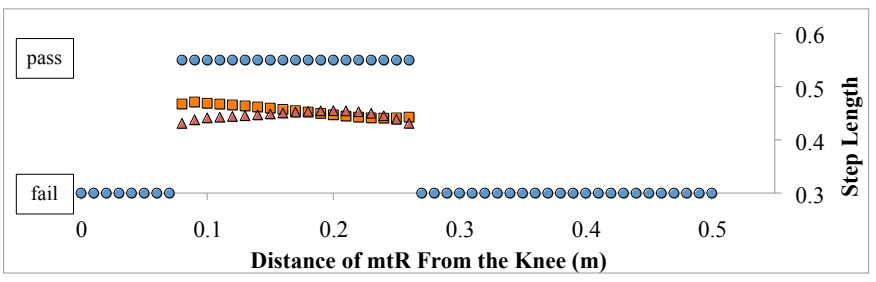

(d) Moving the mass of the right thigh.

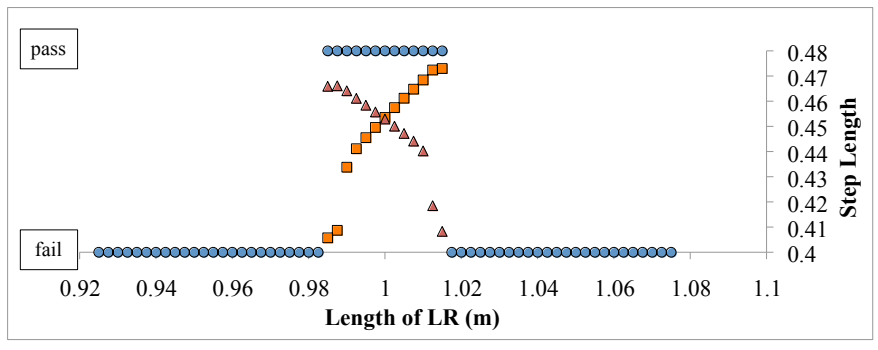

(e) Changing the length of the right leg by changing a1R and b2R.

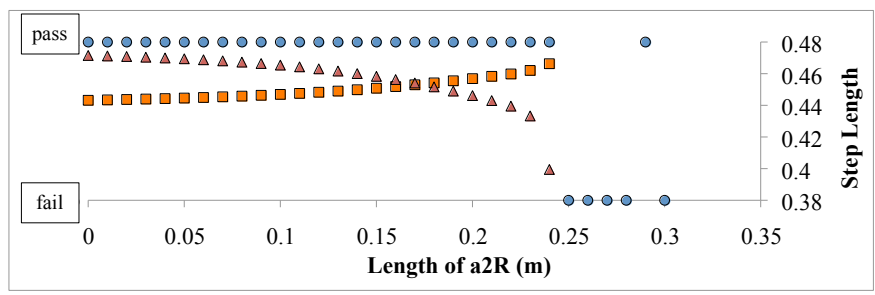

(f) Moving the knee by changing $\mathrm{a} 2 \mathrm{R}$ and $\mathrm{b} 1 \mathrm{R}$ (same leg length).

Pass/Fail

$\triangle$ Right Step Length

Left Step Length

Fig. 7. The success and failure of the different parameters and the step lengths of each leg over all the parameters tested. 


\section{CONClusions AND Future Work}

Our model begins to bridge the gap between recent compass gait models and modern rehabilitation methods. By successfully developing a model with leg specific design parameters, we have created a versatile tool for tuning walking patterns. Furthering our research, we would like to look at each gait pattern presented here and see how it is analogous to an actual human impairment. In doing so, we can further see the merit in each of these patterns and look towards new ways of using them. Eventually, this model may be extended to a three dimensional model, moving ever closer to accurately modeling the human gait. Building a 3-D model would also provide necessary design parameters to successfully build an asymmetric passive dynamic walker. The advantages of both an asymmetric model and a fully developed asymmetric passive dynamic walker are to further test gait rehabilitation methods. Many of these devices focus on mimicking the human neurological control systems, however to do this they need a sound understanding of the dynamic changes. Our model can be used to provide a test bed for developing devices without substantial human interaction. In addition to testing the locations and lengths of parameters, this model can be expanded to include a torsional spring and damper on one or more of the joints, which would mimic spasticity, which is an increased resistance to passive stretch. Looking solely at the dynamics of a passive dynamic walker that is mimicking spasticity, we would also be able to vary the parameters, such as masses, mass locations, and even a torsional spring on the other leg so as to test possible hypotheses about correcting gait.

Another direction for asymmetric gait study can focus on the ability for robots to turn corners. In our attached video, one example for developing an asymmetric gait is changing the position of the knee. Because one leg moves slower than the other, a leg specific single step pattern can be seen. When applied to a walking robot, this pattern could allow a robot to naturally turn a corner or walk a circular path.

\section{ACKNOWLEDGMENTS}

The authors like to thank R. Tedrake for his example MATLAB code.

\section{REFERENCES}

[1] T. McGeer, "Passive Dynamic Walking," The International Journal of Robotics Research, vol. 9, no. 2, pp. 62-82, 1990.

[2] T. D. Sanger, M. R. Delgado, D. Gaebler-Spira, M. Hallett, and J. W. Mink, "Classification and definition of disorders causing hypertonia in childhood," Pediatrics, vol. 111, no. 1, pp. e89-97, 2003.

[3] Y. Otoda, H. Kimura, and K. Takase, "Construction of a gait adaptation model in human split-belt treadmill walking using a two-dimensional biped robot," Advanced Robotics, vol. 23, pp. 535-561, 2009.

[4] R. Margaria, Biomechanics and energetics of muscular exercise. Clarendon Press, 1976.

[5] M. Garcia, A. Chatterjee, A. Ruina, and M. Coleman, "The simplest walking model: Stability, complexity, and scaling," Journal of Biomechanical Engineering, vol. 120, no. 2, p. 281288, 1998.

[6] A. Goswami, B. Thuilot, and B. Espiau, "A Study of the Passive Gait of a Compass-Like Biped Robot," The International Journal of Robotics Research, vol. 17, no. 12, pp. 1282-1301, 1998.

[7] V. F. H. Chen, "Passive Dynamic Walking with Knees: A Point Foot Model," Master's thesis, Massachusetts Institute of Technology, 2005.

[8] S. Collins, A. Ruina, R. Tedrake, and M. Wisse, "Efficient bipedal robots based on passive-dynamic walkers," Science, vol. 307, p. 10821085 , 2005.

[9] A. de Groot, R. Decker, and K. B. Reed, "Gait enhancing mobile shoe (GEMS) for rehabilitation," in Proc. Joint Eurohaptics Conf. and Symp. on Haptic Interfaces for Virtual Environment and Teleoperator Systems, March 2009, pp. 190-195.

[10] I. Handzic and K. B. Reed, "Motion controlled gait enhancing mobile shoe for rehabilitation," in Proc. IEEE Int. Conf. Rehabilitation Robotics, 2011 , in press.

[11] D. Reisman, R. Wityk, K. Silver, and A. Bastian, "Locomotor adaptation on a split-belt treadmill can improve walking symmetry post-stroke," Brain, vol. 130, no. 7, pp. 1861-1872, 2007.

[12] J. T. Choi, E. P. G. Vining, D. S. Reisman, and A. J. Bastian, "Walking flexibility after hemispherectomy: split-belt treadmill adaptation and feedback control." Brain, vol. 132, no. Pt 3, pp. 722-733, Mar 2009.

[13] D. S. Reisman, R. Wityk, K. Silver, and A. J. Bastian, "Splitbelt treadmill adaptation transfers to overground walking in persons poststroke." Neurorehabil Neural Repair, vol. 23, no. 7, pp. 735-744, Sep 2009. 